\title{
Novel risk factors in Cardiovascular disease
}

\section{HMM Herath}

Lecturer in Medicine, Faculty of Medicine, University of Ruhuna, Galle.

\section{Introduction}

The importance of conventional risk factors such as age, smoking, diabetes mellitus, family history of premature heart disease, hypertension, and dyslipidemia in predicting risk of cardiovascular disease (CVD) is well known and these risk factors have been used to predict the future risk of acute myocardial infarction [1,2]. Subsequent advances in understanding the pathogenesis of atherosclerotic vascular disease have stimulated interest in the so-called novel risk factors for CVD. These risk factors are important for 3 reasons.
Firstly, the ability to accurately predict the CVD risk of a specific individual based on his or her conventional risk factor profile is limited. Secondly, the risk prediction using conventional risk factors may not always be accurate as it is not possible to explain all myocardial infarctions. It has been found that only around 50\% case of acute coronary events can be explained with these conventional risk factors. Thirdly, the risk of CVD varies among different ethnic groups, and novel risk factors may partly explain this variation. 
It has been found that inflammation of the endothelium plays an important role in the pathogenesis of atherosclerosis. This new knowledge has lead to the identification of novel risk factors, and the availability of new assessment tools to identify these risk factors. These novel risk factors include lipid variables (small, dense low-density lipoprotein, oxidized low-density lipoprotein, apolipoprotein B), thrombogenic / hemostatic factors (fibrinogen, homocysteine), and inflammatory markers (C-reactive protein) [3].

\section{Highly sensitive C-reactive protein (hsCRP)}

Since atherosclerosis is linked to chronic, low-level inflammation of the vascular endothelium, hsCRP has been utilized to detect this low level of inflammation and thus future risk of atherosclerosis [4,5]. Among currently recognized inflammatory markers, a number of considerations favour hsCRP as a potentially useful predictor of prevalent or incident CVD. Among these are its documented association with cardiovascular risk and the development of a standardized highsensitivity (hs) assay of acceptable cost. Recent studies have shown that hsCRP has independent predictive value of cardiovascular events after adjustment for conventional risk factors [5]. On the contrary, hsCRP has had limited correlation with the severity of atherosclerosis and there are no evidence to show that lowering of CRP lowers CVD risk. However, the American Heart Association had included hsCRP as one of the risk predictor in CVD and the following recommendations were made for the use of hsCRP in clinical practice [6]:

1. hsCRP should be selectively used in primary prevention strategies and it is not recommended for population-wide screening.

2. hsCRP has less clinical utility in secondary prevention because this currently necessitates intensive risk-factor reduction.

3. Test should be done in metabolically stable individuals in the 10\%-20\%, 10-year risk of CVD group according to Framingham predictive instrument. Assessment by conventional risk factors should precede hsCRP testing.
4. hsCRP is not recommended in individual with 10 -year risk of CVD less than $10 \%$ or more than $20 \%$ as the positive results would not change the management of these patients.

5. Two assays, separated by 2 weeks, should be averaged to provide a stable estimate of hsCRP.

6. If the level is $>10 \mathrm{mg} / \mathrm{L}$, a source of overt inflammation should be sought. If found, the value should be discarded and the test repeated after 2 weeks of patient stability.

7. Cutoff points of risk are; Low: $<1.0 \mathrm{mg} / \mathrm{dL}$, average: 1.0-3.0 mg/dL, high: $>3.0 \mathrm{mg} / \mathrm{dL}$.

According to these guidelines it is clear that hsCRP should be used optionally to identify patients without documented CVD but having a $10 \%-20 \% 10$-year risk of CVD and in these individuals further risk stratification by hsCRP would be useful for guiding the intensity of the risk reduction therapy. There are no specific therapies other than intensive risk factor reduction available for the patients with high hsCRP. Therefore, those who are found to have hsCRP levels above $3.0 \mathrm{mg} / \mathrm{dL}$ should be subjected to aggressive life style modification [6].

\section{Homocysteine}

Homocysteine is another novel risk factor which was studied extensively. It is a thiol-containing amino acid intermediate formed during the metabolism of methionine. While atherosclerosis in children with inborn errors of homocysteine metabolism such as cystathionine b-synthase deficiency are associated with markedly elevated plasma homocysteine levels ( $>100 \mu \mathrm{mol} / \mathrm{L})$, it is thought that mild-to-moderate elevations of homocysteine causes atherosclerosis in adults. In the majority of healthy men and women, the serum homocysteine concentration is less than $12 \mu \mathrm{mol} / \mathrm{L}$ and the levels above which has shown to be associated with increased risk of CVD in some studies [7]. The Physicians Health Study found a 3-fold increase in risk of CVD in the individuals with plasma homocysteine levels $12 \%$ above normal. Although this study supports 
hyperhomocysteinemia as a cause of CVD, some other studies failed to demonstrate a clear association [7].

The role of plasma homocysteine in future CVD risk prediction has also not been fully established. This is partly due to the lack of availability of a standardised test to measure homocysteine and uncertainty of the effect of lowering blood homocystine level. Therefore, routine measurement of homocysteine levels was not recommended for purposes of risk assessment, but measurement is optional in patients with earlyonset CVD or in those who develop CVD in the absence of conventional risk factors. If homocysteine levels are elevated, patients should be encouraged to consume the recommended daily intake of folic acid (400 $\mu \mathrm{g} /$ day), as well as vitamins $\mathrm{B}_{6}$ and $\mathrm{B}_{12}$ [8].

\section{Lipoprotein-a [Lp(a)]}

Like LDL cholesterol, Lp(a) is a circulating lipoprotein which has apo B-100 as a surface apolipoprotein. Plasma levels of Lp(a) vary widely among individuals, and generally unrelated to other lipoproteins, and appear to be highly heritable. Risk of cardiovascular events appears to be increased when plasma levels exceed 20 to $30 \mathrm{mg} / \mathrm{dL}$ [9]. However, the Physicians' Health Study and several other recent studies fail to demonstrate such relationship between Lp(a) levels and CVD events $[9,10]$. The lack of evidence along with poor standardization of the test and limited therapeutic options in the treatment of elevated levels have hindered widespread use of $L p(a)$ in CVD risk assessment. Therefore, measurement of $\mathrm{Lp}(\mathrm{a})$ levels is recommended optionally in patients with early-onset CVD or in those who develop CVD in the absence of conventional risk factors. Even though there are no specific pharmacological treatment, aggressive life style modification may reduce $L p(a)$ and is recommended for all patients with elevated Lp(a) [11].

\section{Fibrinogen}

Fibrinogen is an important component of the coagulation pathway, a major determinant of plasma viscosity, and is also an acute phase reactant. Fibrinogen participates in the 2 central processes of atherosclerosis, inflammation and thrombosis, and has been consistently shown to be associated with increased CVD risk. Therefore, fibrinogen screening has the potential to add to CVD risk prediction, but the lack of a standardized assay has limited its use in this context. Whether a reduction in fibrinogen levels will alter clinical outcomes is also currently unknown [12]. Again, no specific therapies are available for elevated fibrinogen, except that in smokers, smoking cessation may reduce fibrinogen concentrations.

\section{Other novel risk factors}

In addition to above novel risk factors, there are other potential risk markers such as oxidized LDL, cell adhesion molecules (ICAM-1), IL-6, TNF-a, asymmetric dimethylarginine (ADMA), apolipoprotein E (Apo E), and serum amyloid A (SAA). However, the routine measurements of these risk factors are currently not recommended due to the poor standardization of the tests and poor correlation with risk prediction $[3,13]$. Few studies found an association between the infection of Chlamydia pneumoniae and CVD events, but subsequent studies failed to demonstrate such association. Therefore, at present marker of such infection would also not be useful.

\section{Summary}

The place of conventional risk factors in primary and secondary prevention of CVD is well established and therefore these risk factors should be aggressively managed. While only a few of the novel risk markers have made their way into clinical use (hsCRP for example), the current era of research suggests that additional risk markers may in the future be available to identify individual risks more precisely and manage them more aggressively.

\section{References}

1. Wilson PW. Established risk factors and coronary artery disease: The Framingham Study. American Journal of Hypertension 1994; 7: 7. 
2. Wilson PW, D’Agostino RB, Levy D. Prediction of coronary heart disease using risk factor categories. Circulation 1998; 97: 1837.

3. Catena C, Cavarape A, Dotto L, et al. The emerging risk factors for CVD: a review of the epidimiologic evidence for lipoprotein (a), homocysteine, and fibrinogen. Advance Clinical Pathology 2003; 7: 3-11.

4. Ridker PM, Brown NJ, Vaughan DE, et al. Established and emerging plasma biomarkers in the prediction of first atherothrombotic events. Circulation 2004; 109: 6-19.

5. Ridker PM. Clinical application of C-reactive protein for cardiovascular disease detection and prevention. Circulation 2003; 107: 363.

6. Pearson TA, Mensah GA, Alexander RW. Markers of inflammation and cardiovascular disease: application to clinical and public health practice: A statement for healthcare professionals from the Centers for Disease Control and Prevention and the American Heart Association. Circulation 2003; 107: 499-511.

7. Kuller LH, Evans RW. Homocysteine, vitamins, and cardiovascular disease. Circulation 1998; 98: 196-9.

8. René M, Malinow MD, Andrew G, Bostom MD, Ronald M, et al. Homocysteine, diet, and cardiovascular diseases: A statement for healthcare professionals from the Nutrition Committee, American Heart Association. Circulation 1999; 99: 178-82.
9. Nguyen TT, Ellefson RD, Hodge DO. Predictive value of electrophoretically detected lipoprotein (a) for coronary heart disease and cerebrovascular disease in a community-based cohort of 9936 men and women. Circulation 1997; 96: 1390.

10. Ridker PM, Hennekens CH, Stampfer MJ. A prospective study of lipoprotein(a) and the risk of myocardial infarction. Journal of American Medical Association 1993; 270: 2195.

11. Seed M, Hoppichler F, Reaveley D, et al. Relation of serum lipoprotein(a) concentration and apolipoprotein (a) phenotype to coronary heart disease in patients with familial hypercholesterolemia. New England Journal of Medicine 1990; 322: 1494.

12. Stec JJ, Silbershatz H, Tofler GH, Matheney TH, Sutherland P, et al. Association of Fibrinogen with Cardiovascular Risk Factors and Cardiovascular Disease in the Framingham offspring population. Circulation 2000; 102: 1634-8.

13. Ridker PM, Rifai N, Stampfer MJ, Hennekens CH. Plasma concentration of interleukin-6 and the risk of future myocardial infarction among apparently healthy Men. Circulation 2000; 101: 1767. 